\title{
Riboswitches: the oldest mechanism for the regulation of gene expression?
}

\author{
Alexey G. Vitreschak ${ }^{1}$, Dimitry A. Rodionov ${ }^{2}$, Andrey A. Mironov ${ }^{2}$ and \\ Mikhail S. Gelfand ${ }^{2}$
}

${ }^{1}$ Institute for Problems of Information Transmission RAS, Bolshoi Karetnyi per. 19, Moscow, 127994, Russia

${ }^{2}$ State Scientific Center GosNIIGenetika, 1st Dorozhnyj proezd 1, Moscow, 113545, Russia

\begin{abstract}
Riboswitches are structures that form in mRNA and regulate gene expression in bacteria. Unlike other known RNA regulatory structures, they are directly bound by small ligands. The mechanism by which gene expression is regulated involves the formation of alternative structures that, in the repressing conformation, cause premature termination of transcription or inhibition of translation initiation. Riboswitches regulate several metabolic pathways including the biosynthesis of vitamins (e.g. riboflavin, thiamin and cobalamin) and the metabolism of methionine, Iysine and purines. Candidate riboswitches have also been observed in archaea and eukaryotes. The taxonomic diversity of genomes containing riboswitches and the diversity of molecular mechanisms of regulation, in addition to the fact that direct interaction of riboswitches with their effectors does not require additional factors, suggest that riboswitches represent one of the oldest regulatory systems.
\end{abstract}

The regulation of gene expression in bacteria involving a choice between two alternative structures in the RNA transcript is a well-known phenomenon [1,2]. In most cases one of the alternative structures (i.e. the repressing conformation) contains a terminator of transcription or a paired region covering the translation initiation site, whereas in the second alternative structure (i.e. the nonrepressing conformation) this regulatory element of the secondary structure is destroyed and the gene(s) are expressed. Which one of these structures will fold depends on the concentration of the target metabolite (e.g. a product of the regulated pathway or a compound to be catabolized) sensed via an intermediate molecule [3]. For example, a regulator protein might bind to one of the structures in the presence of an effector (e.g. TRAP protein [4] or antiterminators of the BglG/SacY family [5]) or uncharged tRNA might stabilize one of the conformations (e.g. T-boxes [6]). In classic attenuators of Escherichia coli, the formation of alternative structures depends on the relative rate of translation by the ribosome (as determined by the concentration of charged tRNAs in attenuators of amino acid operons [1]) and transcription by the RNA polymerase (correlated with the concentration of available nucleotide triphosphates, for example, in the pyrBI operon [7]).
However, recently it was demonstrated that small molecules can bind directly regulatory mRNA structures, called riboswitches [8]. Riboswitches were shown to regulate several metabolic pathways involved in the biosynthesis of vitamins, amino acids and purines. Although the history behind these findings is diverse, common patterns are emerging in all systems that have been studied.

\section{Regulation by riboswitches}

Repression of the riboflavin operon of Bacillus subtilis was initially thought to be due to a regulator protein. However, two candidates for this role, RibC and RibR, which were identified genetically, were later shown to regulate the rib operon indirectly. Both proteins have flavokinase activity and thus decrease the concentration of flavin mononucleotide (FMN), which represses the rib operon [9-11]. The repression was shown to involve premature termination of transcription, generating a short transcript corresponding to the $5^{\prime}$-untranslated leader of the operon mRNA, and to be abolished by mutations in this region [11]. Sequences that could fold into a conserved RNA structure, called the $R F N$-element, were found upstream of several genes involved in riboflavin biosynthesis in a variety of bacterial genomes, and it was suggested that regulation by the $R F N$-element involved direct binding of FMN to mRNA [12]. When more bacterial genome sequences became available, comparative analysis led to the suggestion that the regulatory mechanism is based on the formation of alternative RNA structures, and that the regulation causes premature termination of transcription or inhibition of translation initiation [13]. Both predictions turned out to be correct. Indeed, experiments demonstrated that in B. subtilis, the rib operon is regulated at the level of transcription $[14,15]$, whereas the $y p a A$ gene, positioned elsewhere on the chromosome, is regulated at the level of translation [14]. The secondary structure of the $R F N$-element that was predicted by the comparative analysis was also confirmed.

Similarly, the regulation of thiamin-biosynthetic genes was thought to be mediated by a thiamin pyrophosphate (TPP)-dependent regulatory protein. TPP was later shown to regulate directly translation of the thiCOGE operon in Rhizobium etli via a conserved sequence fragment (thi-box) and a stem-loop mRNA structure overlapping the ribosome-binding site [16]. A more extensive 
structure involving the formation of alternative hairpins (THI-element) was predicted by comparative analysis [17], and observed in experiments in which the structure was shown to regulate translation of the thiM gene in $E$. coli [18], translation and transcription of thiC in E. coli [18] and transcription of the thiamin (tenA) operon in B. subtilis [15].

Another vitamin-related compound, adenosylcobalamin (a derivative of vitamin $\mathrm{B}_{12}$ ), was shown to inhibit ribosome binding to the leader sequence of the btuB gene of E. coli and Salmonella typhimurium [19] and to influence translation of the cob operon of $S$. typhimurium [20]. Direct binding of adenosylcobalamin to the leader region of btuB was demonstrated [21]. Models for the cobalamin-binding structures in the leader regions of these mRNAs were suggested on the basis of chemical probing, mutagenesis and computer prediction $[20,21]$ and enhanced by comparative analysis, leading to the prediction of the B12-element structure [22].

The most recent observations concern the regulation of genes involved in nucleotide and amino acid metabolism. The S-box structure, initially defined by comparative analysis [23], was shown to regulate the transcription of metE, yitJ, $y k r W$ and several other genes in B. subtilis [24-26] and cysH in B. subtilis and Bacillus anthracis [26], after binding to S-adenosylmethionine.

A regulatory mechanism involving an upstream terminator for lysC in B. subtilis has been demonstrated [27]. A lysine riboswitch (L-box or LYS-element) was initially suggested [28] and then studied using comparative genomics $[29,30]$ and in structural mapping experiments [30]. It was shown that in B. subtilis lysine binds to the L-box structure upstream of $l y s C$ [30]. This causes a transition to the structure with a terminator, and the subsequent premature termination of transcription.

Riboswitches regulating various purine biosynthesis and transport genes after binding of purines have been observed in a variety of bacteria and studied experimentally in B. subtilis [28,31], again, despite earlier evidence suggesting the involvement of an unknown protein factor [32]. The purine riboswitch (G-box) regulon in B. subtilis includes pur, xpt-pbuX, pbuG, nupG and pbuE (ydhL) operons [31]. All operons except $y d h L$ are repressed by guanine and hypoxanthine; $y d h L$ is activated [31]. Guanine, xanthine and hypoxanthine, but not adenine, bind to the G-box of $x t p-p b u X$. Finally, binding of adenine to the G-box of $y d h L$ was shown by Winkler et al. [8].

\section{Common features of riboswitches}

To date, at least six RNA elements have been observed to regulate hundreds of genes in many different genomes (e.g. $\sim 2 \%$ of the genome complement of $B$. subtilis are regulated by these RNA elements) [8]. What are common features of riboswitches?

From a biochemical point of view, their most exciting feature is that they all seem to be involved in direct binding by small molecules. In all cases except for the lysine riboswitch the effector ligands contain a nitrogen-containing heterocyclic core (Table 1).

All known riboswitches fold into compact RNA secondary structures with a base stem, a central multiloop and several branching hairpins (Figure 1). The structures were derived by comparative analysis and are supported by complementary substitutions and concur with the available experimental data. The comparison of purine riboswitch structures suggested recently $[28,31]$ illustrates the importance of comparative analysis of RNA structures, as opposed to simple energy minimization methods. Sequence conservation is not confined to paired regions: numerous positions that seem to be single-stranded are also conserved. These positions are likely to be involved in tertiary interactions $[22,29,30]$ and ligand binding.

From the genomic point of view, two striking properties set riboswitches apart from the majority of other regulatory systems. The first feature is the diversity of organisms in which riboswitches have been found (Table 1). The most diverse is the distribution of $T H I$-elements, which have been observed in eubacteria, archaea and eukaryotes. Two other vitamin-related riboswitches, $R F N$ - and $B 12$-elements, were found in the genomes of almost all bacterial taxonomic groups. By contrast, S-boxes, G-boxes and LYS-elements (L-boxes) were observed mainly in Gram-positive bacteria from the Bacillus/Clostridium group, Thermotogale and the Bacteroidetes/Chlorobi group.

The second feature is that riboswitches regulate several different processes (Table 1). The most typical situation, which involves the standard attenuation mechanism, is shown in Figure 2a. In the repressing condition (Figure 2a,i) two RNA structures are formed: the ligand binding stabilizes the riboswitch, and the regulatory hairpin forms. This hairpin can either terminate transcription or inhibit translation initiation by sequestering the ribosome-binding site. In the derepressing condition, the riboswitch is not bound by the ligand and an alternative structure folds. This structure is formed by complementary regions in the riboswitch base stems and part of the regulatory hairpin (Figure 2a,ii,iii). A slight variation occurs when the riboswitch directly sequesters the translation initiation site (Figure $2 b$ ).

If the parity is changed, then the riboswitch is an alternative to the regulatory hairpin (Figure 2c). In this case, gene expression is activated in the presence of the ligand, and repressed in its absence. This type of regulation was demonstrated for purine efflux pump $y d h L$ ( pbuE) [31] and predicted for lysine catabolic operons [29].

It has been suggested that thiamine riboswitches in eukaryotes regulate splicing or mRNA stability [33] but experimental or detailed comparative studies of these systems have not been published yet. Another hypothetical type of regulation by the B12-element and S-box riboswitches is the activation of gene expression via repression of antisense transcripts ([22]; A.G. Vitreschak et al., unpublished). Finally, a complicated secondary structure that is likely to be involved in the activation of translation of human B12-dependent methionine synthase gene by $\mathrm{B}_{12}$ was identified; however, possible involvement of a protein factor was not excluded [34]. This structure has nothing in common with the B12-element, and preliminary analysis shows that it is not conserved upstream of the orthologous rat gene. 
Table 1. Known riboswitches and their properties

\begin{tabular}{|c|c|c|c|c|c|c|}
\hline Riboswitch & Functional system & Ligand & $\begin{array}{l}\text { Preliminary } \\
\text { observations }^{\text {a }}\end{array}$ & $\begin{array}{l}\text { Computer } \\
\text { analysis }^{\text {a }}\end{array}$ & Experiment $^{a}$ & Mechanisms \\
\hline$R F N$-element & $\begin{array}{l}\text { Riboflavin biosynthesis and } \\
\text { transport }\end{array}$ & $\begin{array}{l}\text { FMN (flavin } \\
\text { mononucleotide) }\end{array}$ & [11] & {$[12,13]$} & {$[14,15]$} & $\begin{array}{l}\text { Attenuation of transcription: } \\
\text { Bacillus/Clostridium group, } \\
\text { Thermotogales and Fusobacterium } \\
\text { Inhibition of translation initiation: } \\
\text { Bacillus/Clostridium group (ypaA), } \\
\text { proteobacteria and actinobacteria, } \\
\text { Thermus/Deinococcus group, and } \\
\text { Chloroflexus }\end{array}$ \\
\hline THI-element & $\begin{array}{l}\text { Thiamin biosynthesis; } \\
\text { transport of thiamin and } \\
\text { related compounds }\end{array}$ & $\begin{array}{l}\text { TPP (thiamin } \\
\text { pyrophosphate) }\end{array}$ & [16] & {$[17,33]$} & {$[15,18,33]$} & $\begin{array}{l}\text { Attenuation of transcription: } \\
\text { Bacillus/Clostridium group, } \\
\text { Deinococcus, Thermotogales and } \\
\text { Fusobacterium } \\
\text { Inhibition of translation initiation: } \\
\text { proteobacteria, Bacteroides/Chlorobi } \\
\text { group, Chlorobium } \\
\text { Inhibition of translation initiation } \\
\text { (direct sequestering of the Sd-box) } \\
\text { cyanobacteria, actinobacteria and } \\
\text { Thermoplasma (Archaea) } \\
\text { Dual action: some transporters from } \\
\text { the Bacillus/Clostridium group } \\
3^{\prime} \text {-UTR in plant genes: regulation of } \\
\text { polyadenylation? } \\
\text { Untranslated intron in fungi: regulation } \\
\text { of splicing? }\end{array}$ \\
\hline B12-element & $\begin{array}{l}\text { Cobalamin biosynthesis; } \\
\text { transport of cobalamin and } \\
\text { related compounds; cobalt } \\
\text { transport; cobalamin- } \\
\text { independent isozymes of } \\
\text { cobalamin-dependent } \\
\text { enzymes }\end{array}$ & $\begin{array}{l}\text { Coenzyme B12 } \\
\text { (adenosylcobalamin) }\end{array}$ & {$[19,20]$} & [22] & [21] & $\begin{array}{l}\text { Attenuation of transcription: } \\
\text { Bacillus/Clostridium group, } \\
\text { Thermotogales, Fusobacterium and } \\
\text { Chloroflexus } \\
\text { Inhibition of translation initiation: } \\
\text { proteobacteria, Bacteroides/Chlorobi } \\
\text { group, Deinococcus, actinobacteria } \\
\text { and cyanobacteria }\end{array}$ \\
\hline S-box & $\begin{array}{l}\text { Methionine biosynthesis and } \\
\text { transport; SAM metabolism }\end{array}$ & $\begin{array}{l}\text { SAM (S- } \\
\text { adenosylmethionine) }\end{array}$ & {$[23,51]$} & {$[23,26,34]$} & [24-26] & $\begin{array}{l}\text { Attenuation of transcription: } \\
\text { Bacillus/Clostridium group, Petrotoga } \\
\text { and Chloroflexus } \\
\text { Inhibition of translation initiation: } \\
\text { Bacteroidetes/Chlorobi group } \\
\text { Inhibition of translation initiation } \\
\text { (direct sequestering of the Sd-box): } \\
\text { actinobacteria } \\
\text { Unknown: Deinococcus, Xanthomonas } \\
\text { and Geobacter }\end{array}$ \\
\hline $\begin{array}{l}\text { G-box or } \\
\text { XptR regulon }\end{array}$ & $\begin{array}{l}\text { Purine metabolism and } \\
\text { transport }\end{array}$ & Purines & [32] & {$[29,31]$} & {$[29,31]$} & $\begin{array}{l}\text { Attenuation of transcription: } \\
\text { Bacillus/Clostridium groups, } \\
\text { Fusobacterium } \\
\text { Attenuation of transcription (inhibition } \\
\text { of premature termination): purine } \\
\text { efflux transporter of Bacillus subtilis } \\
\text { Unknown: Vibrio vulnificus }\end{array}$ \\
\hline $\begin{array}{l}\text { L-box or } \\
\text { LYS-element }\end{array}$ & $\begin{array}{l}\text { Lysine biosynthesis, transport } \\
\text { and catabolism }\end{array}$ & Lysine & {$[27,28]$} & {$[28,30]$} & [30] & $\begin{array}{l}\text { Attenuation of transcription: } \\
\text { Bacillus/Clostridium group and } \\
\text { Thermotogales } \\
\text { Inhibition of translation initiation: } \gamma \text { - } \\
\text { proteobacteria (i.e. Enterobacteriae, } \\
\text { Pasteurellaceae, Vibrionaceae and } \\
\text { Shewanella) } \\
\text { Attenuation of transcription (inhibition } \\
\text { of premature termination): Iysine } \\
\text { utilization operons of } \\
\text { Thermoanaerobacter and } \\
\text { Fusobacterium }\end{array}$ \\
\hline
\end{tabular}

${ }^{\mathrm{a}}$ Futher details can be obtained from the references listed in the table.

\section{Evolution of riboswitches}

Notably, there is a correlation between the preferred mechanism of regulation and taxonomy. Thus, Grampositive bacteria from the Bacillus/Clostridium group, Thermotogales and Fusobacterium tend to have riboswitches that act as attenuators of transcription, whereas proteobacteria usually have riboswitches that control the initiation of translation. This is not an absolutely universal rule because it does not take into account cases of dual regulation (e.g. the $y p a A$ genes from the Bacillus/Clostridium group, which are regulated both on the transcriptional level [35] and on the translational [14] level). This correlation can also be obscured by horizontal transfer, when a gene or an operon together with the regulating riboswitch is transferred between taxa. In addition to overall similarity, many riboswitches have taxon-specific sequence and taxon-specific structural features $[13,22]$.

In addition to the normal vertical descent, the evolution of riboswitches involved numerous genome-specific duplications and horizontal transfers. All these events are easily discernible in phylogenetic trees. Orthologous riboswitch elements evolving by speciation form branches corresponding to orthologous genes that are regulated by 


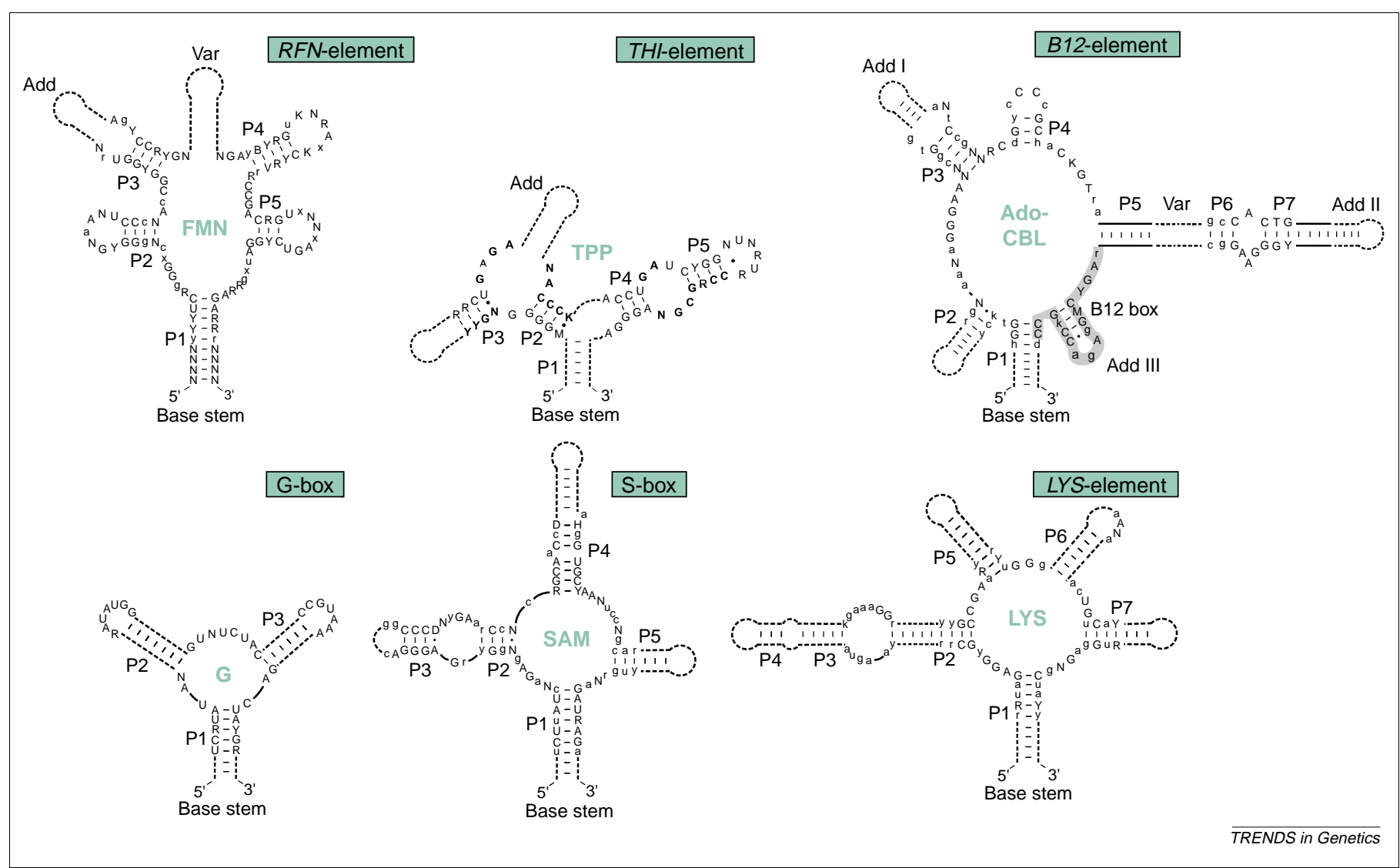

Figure 1. Riboswitch structures. The structures of riboswitches contain conserved base-paired regions and invariant (uppercase) and highly conserved (lowercase) positions, although some parts of the structures are variable (Var) or facultative (Add). The conserved helices are numbered independently P1 through P7, P1 being the base stem; regions identified in early experiments (thi-element and B12-element) are highlighted in bold and in gray, respectively. Abbreviations: Ado-CBL, adenysylcobalamin, FMN, flavin mononucleotide; G, guanine; LYS, lysine; SAM, S-adenosylmethionine; TPP, thiamin pyrophosphate.

these riboswitches. Recently duplicated elements form small genome-specific branches; many examples of these branches can be seen in the B12-element tree [22]. Notably, the same duplication might have happened independently on several occasions. For example, $R F N$-elements of the $r i b$-operons and genes ypaA are closely related in the genomes of Staphylococcus aureus and Clostridium spp. [13], whereas B12-elements of btuR and btuF are similar in Chloroflexus aurantiacus and Thermoanaerobacter tengcongensis [22].

Horizontal transfer is evident when a phylogenetic tree contains a cluster of riboswitches corresponding to orthologous genes from distant bacteria; more exactly, a riboswitch is embedded in a cluster of riboswitches regulating the same gene in distant genomes. Additional support for these observations is provided by the phylogenetic trees of genes. Often the gene regulated by a riboswitch belongs to the branch formed by the genes regulated by riboswitches from the 'recipient' cluster, and thus the gene tree shows the same signs of horizontal transfer.

An interesting example is the riboflavin operon of two $\gamma$-proteobacteria from the Pasteurallaceae family, Haemophilus ducreyi and Actinobacillus pleuropneumoniae, that has been transferred, together with the upstream $R F N$-element, from some Gram-positive bacteria belonging to the Bacillus/Clostridium group [13]. Similarly, the $c b i$ operon of enterobacteria, together with the upstream
B12-element, probably originates in the Bacillus/Clostridium group [22]. A short-distance transfer was observed in Pseudomonas spp.: a $R F N$-element upstream of ribE2 gene belonging to the candidate riboflavin operon of Pseudomonas aeruginosa is closely related to the $R F N$-elements of single ribH2 genes in other pseudomonads [13]. Because riboswitch-based regulation does not require any additional proteins or genome-specific finely tuned factors, such as translation and transcription rates, riboswitches should be highly amenable to horizontal transfer. Therefore, one might argue that THI-elements of archeae are horizontally transferred from eubacteria. This is supported by the fact that archaeal THI-elements are restricted to one narrow taxonomic group, Thermoplasma spp. However, the genes that are regulated by $T H I$-elements, the candidate transporters thiT, are also taxon-specific [17].

\section{Riboswitches and other regulatory systems}

Taken separately, these properties are not unique for the riboswitch systems. Aptamers, RNA structures that can directly interact with small molecules, have been generated by in vitro selection for a wide range of ligands [36,37]. Moreover, an artificial regulatory construct involving an aptamer structure was shown to be effective in vivo: the addition of the aptamer-binding compound stabilized a hairpin that interfered with initiation of translation of a reporter gene [38]. It is possible to use aptamers for the regulation of cellular processes, such as the cell cycle [39]. 


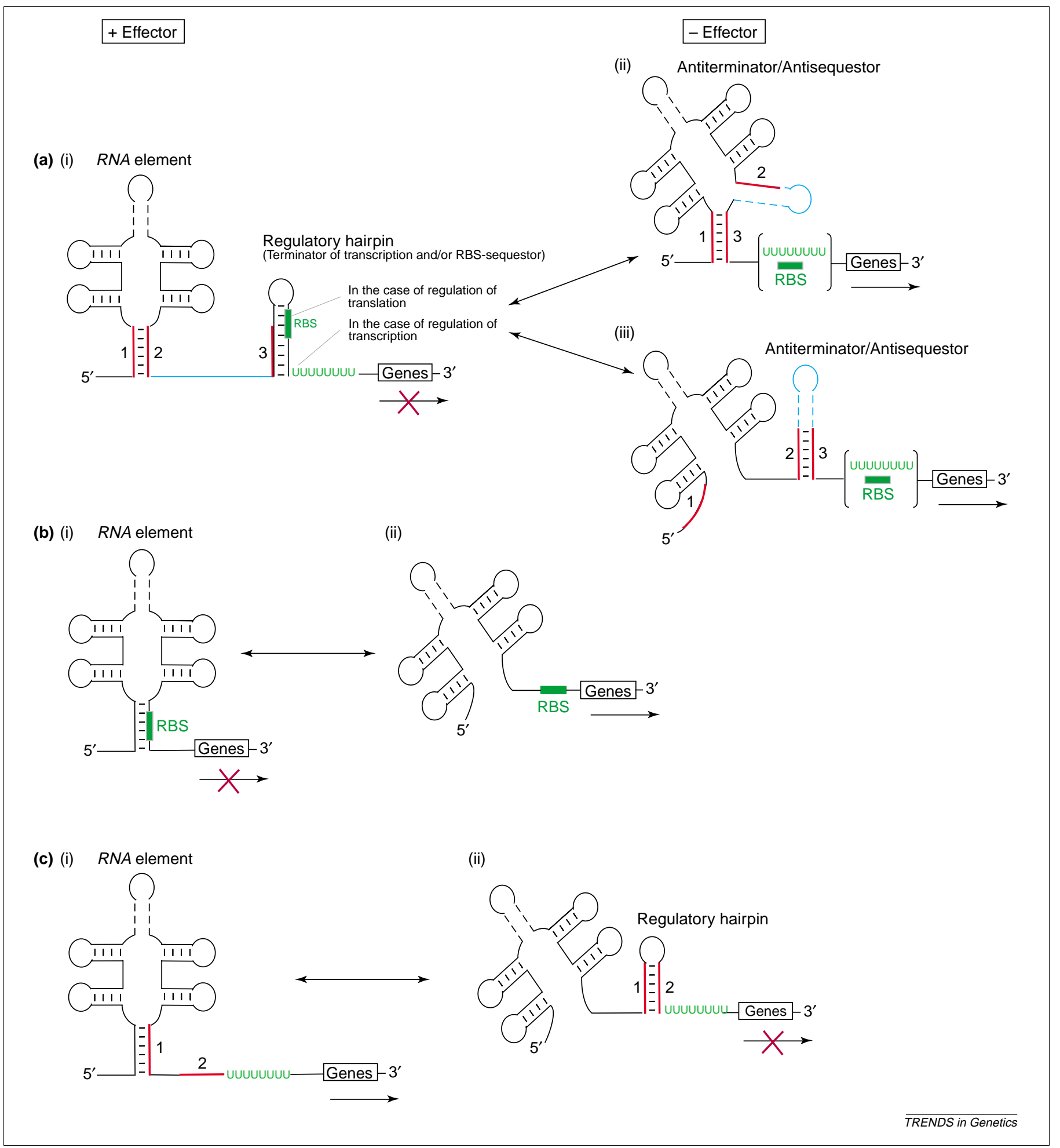

Figure 2. The mechanisms of the riboswitch regulation involve the formation of alternative structures. The effector stabilizes the repressing conformation in (a)(i) and (b)(i), whereas in (c)(ii) the repressing conformation folds in the absence of the effector. (a) Attenuation of transcription [via premature termination, (ii)] or translation [via inhibition of initiation, (iii)]. (b) Attenuation of translation involves the direct sequestering of the translation initiation site. (c) Activation via inhibition of premature termination of transcription. The regions involved in alternative interactions are shown in red. Regulatory elements, such as ribosome binding sites (RBS) or poly- $U$ tracts in terminators are shown in green.

Functional similarity between aptamers and riboswitches has been suggested [40]. It should be noted however that there is no obvious similarity between G-boxes, $R F N$ - and B12-elements and known aptamers for xanthine and guanine [41], FMN [42] and cyanocobalamin (vitamin $\mathrm{B}_{12}$ ) [43], respectively.
Common regulatory signals exist in bacteria and archea, and in bacteria and eukaryotes. In particular, the transcriptional regulator BirA has the same recognition signal in eubacteria and archaea [44], whereas BARBIE-box regulates the transcription of phenobarbitalinduced genes in Bacillus megaterium, insects and 
mammals [45]. One more example is given by the selenocystein insertion sequence (SECIS) RNA elements that guide the incorporation of selenocysteine in bacterial, archaeal and eukaryotic selenoproteins [46]. Notably, in bacteria SECIS hairpins occur immediately downstream of the selenocysteine UGA codons, whereas in archaea and eukaryotes they are positioned in $3^{\prime}$ unstranslated regions. Thus, the mechanism of their action might be different.

The diversity of functional mechanisms also has been observed in other regulatory systems. For example, TRAP, a regulator of tryptophan metabolism in Gram-positive bacteria, is involved in transcription attenuation of the $\operatorname{trpEDCFBA}$ operon, translation attenuation of the proximal gene of the operon, $\operatorname{trpE}$ (via a Shine-Dalgarno-box sequestering hairpin) and translation attenuation of $\operatorname{trp} G$ (via direct binding to the translation initiation site) [4].

Taken together, the properties of riboswitches illustrate their ancient origin. Their wide distribution, combination of generic and taxon-specific structural elements, and, most importantly, independence of trans-acting protein factors make them ideal candidates for the role of regulators in the RNA world. Their high structural conservation can be explained by strong functional pressure: minor differences in S-boxes of cysH in B. subtilis and $B$. anthracis lead to a two order of magnitude difference in affinity towards SAM [25].

Many functional systems regulated by riboswitches also have other modes of regulation. S-boxes are replaced by methionine-specific T-boxes in Lactobacilli and by transcriptional factors in Streptococci and $\gamma$-proteobacteria (A.G. Vitreschak et al., unpublished). Cobalamin metabolism is likely to be regulated by a transcription factor in some archaea [47]. Purine metabolism is regulated by (unrelated) repressors of transcription PurR in bacteria from the Bacillus/Clostridium group [48] and in $\gamma$-proteobacteria [49]. Moreover, lysine metabolism in $\gamma$-proteobacteria is regulated not only by $L Y S$-elements but also by the LysR repressor [50]. Finally, in B. subtilis, $x p t-p b u X$ and $p b u G$ are under dual regulation by purine riboswitches and PurR [31,48].

If the hypothesis of the ancient origin of riboswitches is correct, these other systems are relative newcomers and it should be interesting to study the evolution of old and new regulatory systems of the same metabolic pathways. Because riboswitches are highly conserved, they are an ideal tool for metabolic reconstruction and computational annotation of gene function, particularly in less-studied genomes, leading to the identification of numerous missing enzymes and the assignment of specificity to dozens of transporters ([13,17,29,30,31,47]; A.G. Vitreschak et al., unpublished). Finally, there are no reasons to believe that the riboswitch family is restricted to the six known cases, and it is likely that it will increase as more genomes and functional systems are analyzed.

\section{References}

1 Landick, R. et al. (1996) Transcription attenuation. In Escherichia coli and Salmonella: Cellular and Molecular Biology (Vol. 1) (Neidhardt, F.C., ed.), pp. 1263-1286, ASM Press

2 Merino, E. and Yanofsky, C. (2002) Regulation by terminationantitermination: a genomic approach. In Bacillus subtilis and its
Closest Relatives: From Genes to Cells (Sonenshein, A.L., ed.), pp. 323-336, ASM Press

3 Henkin, T.M. and Yanofsky, C. (2002) Regulation of transcription attenuation in bacteria: how RNA provides instructions for transcription termination/antitermination decisions. Bioessays 24, 700-707

4 Babitzke, P. (1997) Regulation of tryptophan biosynthesis: Trp-ing the TRAP or how Bacillus subtilis reinvented the wheel. Mol. Microbiol. $26,1-9$

5 Aymerich, S. and Steinmetz, M. (1992) Specificity determinants and structural features in the RNA target of the bacterial antiterminator proteins of the BglG/SacY family. Proc. Natl. Acad. Sci. U. S. A. 89, 10410-10414

6 Grundy, F.J. et al. (2002) Sequence requirements for terminators and antiterminators in the $\mathrm{T}$ box transcription antitermination system: disparity between conservation and functional requirements. Nucleic Acids Res. 30, 1646-1655

7 Roland, K.L. et al. (1988) Role of the ribosome in suppressing transcriptional termination at the pyrBI attenuator of Escherichia coli K-12. Proc. Natl. Acad. Sci. U. S. A. 85, 7149-7153

8 Winkler, W.C. and Breaker, R.R. (2003) Genetic control by metabolitebinding riboswitches. Chembiochem 4, 1024-1032

9 Mack, M. et al. (1998) Regulation of riboflavin biosynthesis in Bacillus subtilis is affected by the activity of the flavokinase/flavin adenine dinucleotide synthase encoded by ribC. J. Bacteriol. 180, 950-955

10 Solovieva, I.M. et al. (1999) The ribR gene encodes a monofunctional riboflavin kinase which is involved in regulation of the Bacillus subtilis riboflavin operon. Microbiology 145, 67-73

11 Lee, J.M. et al. (2001) RNA expression analysis using an antisense Bacillus subtilis genome array. J. Bacteriol. 183, 7371-7380

12 Gelfand, M.S. et al. (1999) A conserved RNA structure element involved in the regulation of bacterial riboflavin synthesis genes. Trends Genet. 15, 439-442

13 Vitreschak, A.G. et al. (2002) Regulation of riboflavin biosynthesis and transport genes in bacteria by transcriptional and translational attenuation. Nucleic Acids Res. 30, 3141-3151

14 Winkler, W.C. et al. (2002) An mRNA structure that controls gene expression by binding FMN. Proc. Natl. Acad. Sci. U. S. A. 99, 15908-15913

15 Mironov, A.S. et al. (2002) Sensing small molecules by nascent RNA: a mechanism to control transcription in bacteria. Cell 111, 747-756

16 Miranda-Rios, J. et al. (2001) A conserved RNA structure (thi box) is involved in regulation of thiamin biosynthetic gene expression in bacteria. Proc. Natl. Acad. Sci. U. S. A. 98, 9736-9741

17 Rodionov, D.A. et al. (2002) Comparative genomics of thiamin biosynthesis in procaryotes. New genes and regulatory mechanisms. J. Biol. Chem. 277, 48949-48959

18 Winkler, W. et al. (2002) Thiamine derivatives bind messenger RNAs directly to regulate bacterial gene expression. Nature 419, 952-956

19 Nou, X. and Kadner, R.J. (2000) Adenosylcobalamin inhibits ribosome binding to btuB RNA. Proc. Natl. Acad. Sci. U. S. A. 97, 7190-7195

20 Ravnum, S. and Andersson, D.I. (2001) An adenosyl-cobalamin (coenzyme-B12)-repressed translational enhancer in the $c o b$ mRNA of Salmonella typhimurium. Mol. Microbiol. 39, 1585-1594

21 Nahvi, A. et al. (2002) Genetic control by a metabolite binding mRNA. Chem. Biol. 9, 1043-1049

22 Vitreschak, A.G. et al. (2003) Regulation of the vitamin B12 metabolism transport in bacteria by a conserved RNA structural element. RNA 9, 1084-1097

23 Grundy, F.J. and Henkin, T.M. (1998) The S box regulon: a new global transcription termination control system for methionine and cysteine biosynthesis genes in gram-positive bacteria. Mol. Microbiol. 30, 737-749

24 Epshtein, V. et al. (2003) The riboswitch-mediated control of sulfur metabolism in bacteria. Proc. Natl. Acad. Sci. U. S. A. 100, 5052-5056

25 McDaniel, B.A. et al. (2003) Transcription termination control of the $\mathrm{S}$ box system: direct measurement of S-adenosylmethionine by the leader RNA. Proc. Natl. Acad. Sci. U. S. A. 100, 3083-3088

26 Winkler, W.C. et al. (2003) An mRNA structure that controls gene expression by binding S-adenosylmethionine. Nat. Struct. Biol. 10, 701-707

27 Kochhar, S. and Paulus, H. (1996) Lysine-induced premature transcription termination in the lysC operon of Bacillus subtilis. Microbiology 142, 1635-1639 
28 Mandal, M. et al. (2003) Riboswitches control fundamental pathways in Bacillus subtilis and other bacteria. Cell 113, 577-586

29 Rodionov, D.A. et al. Regulation of lysine biosynthesis and transport in bacteria: yet another riboswitch. Nucleic Acids Res. (in press)

30 Grundy, F.J. et al. (2003) The L box regulon: Lysine sensing by leader RNAs of bacterial lysine biosynthesis genes. Proc. Natl. Acad. Sci. U. S. A. $100,12057-12062$

31 Johansen, L.E. et al. (2003) Definition of a second Bacillus subtilis pur regulon comprising the pur and $x p t-p b u X$ operons plus $p b u G$, nupG (yxjA), and pbuE (ydhL). J. Bacteriol. 185, 5200-5209

32 Christiansen, L.C. et al. (1997) Xanthine metabolism in Bacillus subtilis: characterization of the xpt-pbuX operon and evidence for purine- and nitrogen-controlled expression of genes involved in xanthine salvage and catabolism. J. Bacteriol. 179, 2540-2550

33 Sudarsan, N. et al. (2003) Metabolite-binding RNA domains are present in the genes of eukaryotes. RNA 9, 644-647

34 Oltean, S. and Banerjee, R. (2003) Nutritional modulation of gene expression and homocysteine utilization by vitamin B12. J. Biol. Chem. 278, 20778-20784

35 Lee, J.M. et al. (2001) RNA expression analysis using an antisense Bacillus subtilis genome array. J. Bacteriol. 183, 7371-7380

36 Hermann, T. and Patel, D.J. (2000) Adaptive recognition by nucleic acid aptamers. Science 287, 820-825

37 Famulok, M. (1999) Oligonucleotide aptamers that recognize small molecules. Curr. Opin. Struct. Biol. 9, 324-329

38 Werstuck, G. and Green, M.R. (1998) Controlling gene expression in living cells through small molecule-RNA interactions. Science 282, $296-298$

39 Grate, D. and Wilson, C. (2001) Inducible regulation of the S. cerevisiae cell cycle mediated by an RNA aptamer-ligand complex. Bioorg. Med. Chem. 9, 2565-2570

40 Stormo, G.D. and Ji, L. (2001) Do mRNA act as direct sensors of small molecules to control their expression? Proc. Natl. Acad. Sci. U. S. A. 98, 9465-9467

41 Kiga, D. et al. (1998) An RNA aptamer to the xanthine/guanine base with a distinctive mode of purine recognition. Nucleic Acids Res. 26, $1755-1760$

42 Fan, P. et al. (1996) Molecular recognition in the FMN-RNA aptamer complex. J. Mol. Biol. 258, 480-500

43 Sussman, D. et al. (2000) The structural basis for molecular recognition by the vitamin B12 RNA aptamer. Nat. Struct. Biol. 7, 53-57

44 Rodionov, D.A. et al. (2002) Conservation of the biotin regulon and the BirA regulatory signal in eubacteria and archaea. Genome Res. 12, $1507-1516$

45 Gelfand, M.S. (1999) Recognition of regulatory sites by genomic comparison. Res. Microbiol. 150, 755-771

46 Krol, A. (2002) Evolutionarily different RNA motifs and RNA-protein complexes to achieve selenoprotein synthesis. Biochimie 84, 765-774

47 Rodionov, D.A. et al. (2003) Comparative genomics of the vitamin B12 metabolism and regulation in prokaryotes. J. Biol. Chem. 278, 41148-41159

48 Saxild, H.H. et al. (2001) Definition of the Bacillus subtilis PurR operator using genetic and bioinformatic tools and expansion of the

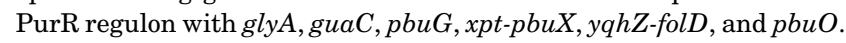
J. Bacteriol. 183, 6175-6183

49 Zalkin, H. and Nygaard, P. (1996) Biosynthesis of purine nucleotides. In Escherichia coli and Salmonella: Cellular and Molecular Biology (Vol. 1) (Neidhardt, F.C., ed.), pp. 561-579, ASM Press

50 Patte, J-C. (1996) Biosynthesis of threonine and lysine. In Escherichia coli and Salmonella: Cellular and Molecular Biology (Vol. 1) (Neidhardt, F.C., ed.), pp. 528-541, ASM Press

51 Auger, S. et al. (2002) The metIC operon involved in methionine biosynthesis in Bacillus subtilis is controlled by transcription antitermination. Microbiology 148, 507-518

\section{TiGS online - making the most of your personal subscription}

High-quality printouts (from PDF files)

Links to other articles, other journals and cited software and databases

$$
\text { All you have to do is: }
$$

Obtain your subscription key from the address label of your print subscription.

Then go to http://www.trends.com, click on the Claim online access button and select

Trends in Genetics.

You will see a BioMedNet login screen.

Enter your BioMedNet username and password. If you are not already a BioMedNet member, please click on the Register button. Once registered, you will be asked to enter your subscription key. Following confirmation, you will have full access to

$$
\text { Trends in Genetics. }
$$

If you obtain an error message please contact Customer Services (info@current-trends.com) stating your subscription key and BioMedNet username and password. Please note that you only need to enter your subscription key once; BioMedNet 'remembers' your subscription. Institutional online access is available at a premium. If your institute is interested in subscribing to print and online, please ask them to contact ct.subs@qss-ik.com 